\title{
Evaluating the Regional Benefit/Cost Ratio for Transit State of Good Repair Investments
}

\author{
Liz Paterson \\ UN-HABITAT \\ David Vautin \\ Metropolitan Transportation Commission (San Francisco Bay Area)
}

\begin{abstract}
Should transit operators focus scarce funding on maintaining current systems in a state of good repair (SGR), or on expanding transit systems? Prior to this analysis, user impacts of transit SGR had not been systematically calculated. This study develops a new methodology for assessing the impacts of SGR on ridership, vehicle miles traveled, travel times and costs, and public health and safety. This is done for the 25 major transit systems in the nine-county San Francisco Bay Area. Moreover, the study uses a methodology parallel to that used to assess transit system expansion in the Bay Area and, therefore, is able to compare the benefit/cost ratios of transit expansion vs. transit SGR on an even footing. Results indicate regional benefit/cost ratios of close to 3 for transit SGR, with diminishing returns at higher funding levels. This is similar to the benefit/cost ratio of the average transit expansion project.
\end{abstract}

\section{Background}

In the San Francisco Bay Area and cities throughout the United States, there is an ongoing debate about the best use of transit funding. Some argue that maintaining current assets in a state of good repair (SGR) should take priority over expanding transit systems. Others argue that cities and regions need to continue expanding their transit network to enable modal shift in underserved communities, a strategy that can come at the expense of system preservation without an influx of additional funds.

The nine-county San Francisco Bay Area metropolitan planning organization-known as the Metropolitan Transportation Commission (MTC) - completes a rigorous performance assessment for expansion projects and operational improvement projects as part of the regional planning process (Metropolitan Transportation Commission 2013). Projects proposed for inclusion in the regional transportation plan (RTP) are 
evaluated for their cost-effectiveness using a model-based methodology to calculate a benefit/cost $(B / C)$ ratio. However, this methodology has been used only to examine the benefits of expansion projects and operational changes; there is no existing methodology to assess user and regional benefits of transit SGR. In fact, there has never been published research quantitatively linking transit SGR with ridership, a key component in a regional benefit/cost assessment.

This study defines a new methodology to link transit state of good repair with impacts on ridership and regional benefits as a whole, piloting this methodology with the 25 major transit systems in the San Francisco Bay Area. The results of this analysis provide a benefit/cost ratio for transit SGR funding. This ratio can be compared on an equal footing with the $B / C$ ratio transit expansion projects assessed as part of the most recent Regional Transportation Plan (RTP), "Plan Bay Area."

\section{Literature Review}

Efforts to quantify benefits of transit state of good repair generally have stopped short of linking asset condition with user impacts or ridership. It has been demonstrated that poorly-maintained transit systems can experience large ridership reductions based on the experience of rail systems in New York, Chicago, and Philadelphia in the 1970s and 1980s (Deakin et al. 2012). However, these studies do not systematically quantify the relationship between SGR spending and user benefits. Furthermore, the link between transit asset management and user impacts has yet to be modeled using a regional travel demand model to understand systemwide and multimodal impacts beyond riders.

A study by the U.S. Government Accountability Office finds that, although transit agencies sometimes track SGR backlog and on-time service, none of the agencies link SGR to future ridership. The report suggests that understanding the implications of SGR on ridership could help transit agencies optimize their asset management strategies (U.S. GAO 2013).

Another recent report by the Transit Cooperative Research Program, State of Good Repair: Prioritizing the Rehabilitation and Replacement of Existing Capital Assets and Evaluating the Implications for Transit (TCRP Report 157), includes a comprehensive literature review of transit asset management practices. The report finds that programs across the country generally rely upon asset ages to determine predicted condition and replacement needs. The only system currently tying asset condition to user impacts is the London Underground. Unfortunately, this methodology has not yet been published (Transportation Research Board 2012).

Perhaps the most powerful and widely-used transit asset management software is the Federal Transit Administration's (FTA) Transit Economic Requirements Model (TERM) and its counterpart for local- and regional-level analysis, TERM-Lite. However, as highlighted by a broad review of TERM by Cohen (2014), the software tracks asset age without linking it to system performance or public benefits. Cohen proposes that a useful addition to TERM's capabilities would develop and use a model to quantitatively link failures to total passenger delay, building upon the TCRP 157 framework. 
There are two exceptions to the dearth of studies linking transit SGR and user impacts. One is a 2012 regional impacts study examining SGR investments into the San Francisco Bay Area's heavy rail system known as BART (Deakin et al. 2012). The study estimates user impacts based on some broad assumptions that are very problematic; however, the report includes useful data from focus group interviews, which found that travel times and costs are the primary factors in transit mode choice. Only non-riders noted that crime, cleanliness, and noise would deter them from taking BART, indicating that deterioration of these elements would likely have small impacts on ridership.

The other study that links transit SGR with broad impacts in a recent study of the Southern Pennsylvania Transportation Authority (SEPTA) (Voith, Angelides, and Ozimek 2013). Results of econometric modeling indicate that completely eliminating SEPTA would increase costs to travelers by $\$ 488$ million annually, cause externalities associated with higher automobile usage, reduce public revenues and property values, and trigger the loss of 60,000 jobs. Importantly, the authors note that they examine the extreme case of complete transit elimination partly because they do not have the means to simulate the incremental reduction in services that would result from a less-than-full capital shortfall: "A concrete analysis of economic impacts associated with underfunding SEPTA's capital needs would require a direct connection between the extent to which the capital shortfall will result in reduced transit services, then use those specific changes in service patterns to model the impact on ridership and congestion" (p. 15). The current study fills this gap.

Our study builds upon existing research by quantifying the linkages between asset ages, failure rates, delay, ridership, and broader regional impacts for 25 of the Bay Area's transit systems. We focused on delay as the primary operational impact of transit asset failure based on the results of the BART focus group interviews. We assumed that transit operators will hold ticket prices constant in various SGR scenarios. While passenger experiences of comfort, cleanliness, and safety may have an impact on travel behavior, Cohen notes that there is a lack of analytical procedures for relating asset age to passenger comfort (Cohen 2014). In the present study, we were able to answer Cohen's call to link transit asset management best practices with user impacts. We believe this gives the best and most detailed estimation yet of the regional impacts of funding for transit state of good repair.

\section{Methodology}

To predict regional benefits for transit SGR funding scenarios, we calculated travel delays associated with aging transit assets and used those as inputs into the Bay Area's regional activity-based travel model (Travel Model One) in the form of in-vehicle and

\footnotetext{
${ }^{1}$ The study assumes that an SGR funding shortfall affects all asset categories equally, whereas, in reality, funding sources and operators prioritize assets for funding based on their impact on system operations. Second, the authors predict ridership reductions directly corresponding with projected decreases in train capacity (assuming older trains have declining availability) and reduce predicted ridership further due to delays and discomfort. However, because BART trains generally are not currently full to capacity, capacity reductions likely will not translate directly into ridership reductions. Additionally, the study does not specify formulas for translating asset age into reported delays and asset failures.
} 
out-of-vehicle travel times. We focused on travel time instead of cost or safety for the following reasons: (1) the cost of transit to users is determined by operators and not directly dependent upon SGR maintenance funding, and (2) safety risks generally are dealt with by instituting slow zones or removing assets from operation, actions that counted as a "failure" in our model and thus contribute to delays (Cohen 2014).

Travel Model One simulates travel behavior for a typical workday. In this context, we could not simulate location-specific failures that occur less than once daily. Additionally the Bay Area's Regional Transit Capital Inventory (RTCI), which tracks all transit assets, does not yet contain locational information. For these reasons, we calculated average delay (based on probability) occurring when the average asset (by type) in the average location fails for each operator and mode. We then added this expected delay to all of the operator's routes of that mode. This effectively served a proxy for system reliability due to the level of system maintenance. Figure 1 summarizes the approach taken to link funding scenarios, travel times, and regional benefits.

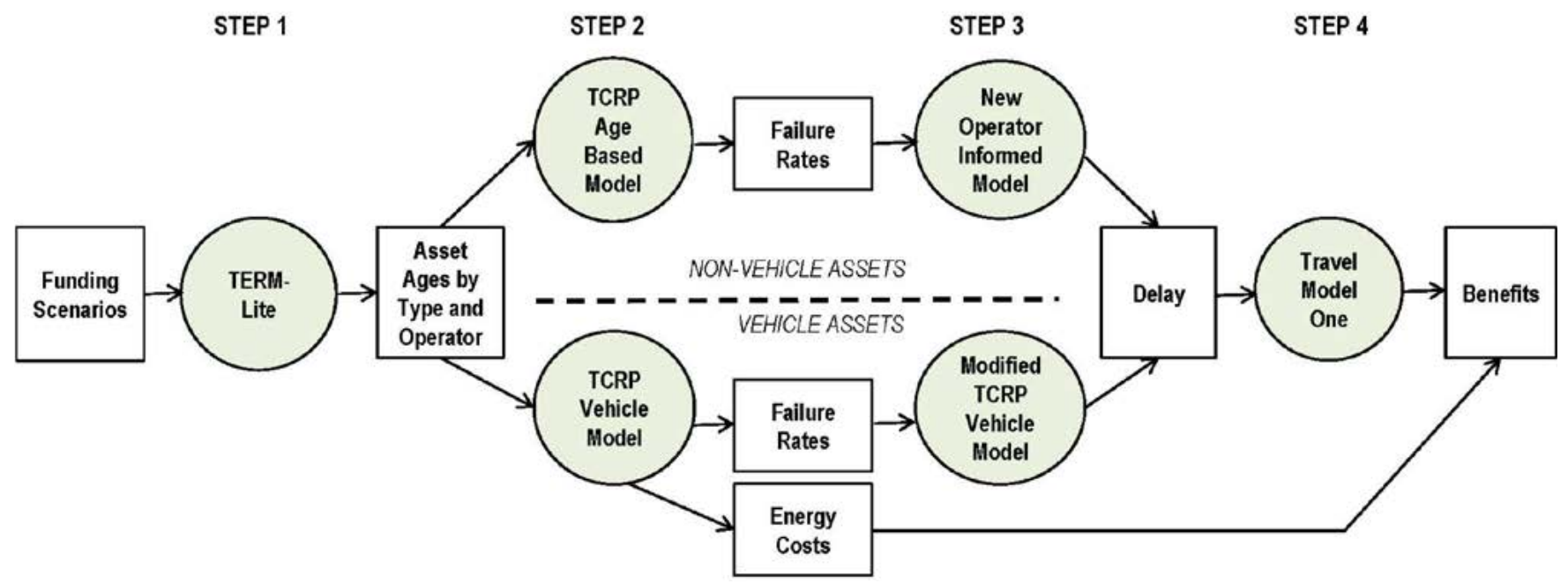

FIGURE 1. Pathway between funding scenarios and benefit calculations for transit SGR

\section{Step 1: Link Funding Scenarios with Asset Conditions using TERM-Lite Model}

MTC's RTCl is used in conjunction with TERM-Lite to help prioritize the allocation of funding to be used for maintenance, rehab, and replacement of transit assets. Under a given funding scenario or a backlog target for a future year, the TERM-Lite model can calculate the age of each transit asset in the RTCI for a future year. We used TERM-Lite to approximate the replacements made by system operators in each year to predict asset ages in year 2040.

Each SGR funding scenario was compared to a baseline of current conditions. This led to cases in which both benefits and costs were negative (i.e., cases of spending less than is necessary to achieve baseline conditions and getting fewer benefits). Benefit/cost ratios for such degradation scenarios can be seen as representing the cost-effectiveness of moving from a funding level below baseline to the baseline funding level. 


\section{Step 2 (Vehicles): Link Vehicle Ages with Failure Rates and Energy Costs Using TCRP's Vehicle Model}

TCRP's Vehicle Model (Transportation Research Board 2012) provides an equation for linking a vehicle's lifetime miles with energy costs per vehicle mile. We used this equation to predict energy consumption costs in 2040 based on vehicle ages. To do this, we estimated lifetime mileage based on age using a constant for average annual mileage by operator and asset. Base-year energy costs per mile for 2040 were calculated using standard MTC projections for year 2040. Then, the TCRP model was applied to each transit vehicle in the RTCl. Average energy costs per mile for each operator and vehicle were then used to calculate total projected energy costs for each operator in 2040, drawing upon outputs from Travel Model One, which show how many miles are traveled by each transit operator in 2040. The difference between total scenario energy costs and baseline scenario energy costs for each operator was subtracted from the benefits side of the benefit/cost ratio. This reflects additional energy costs due to aging vehicles in a given scenario.

TCRP's Vehicle Model also provides an equation for linking bus and train ages with road calls or vehicle failures per mile. We used this equation with data on base-year failures by operator and mode previously collected by MTC and the age of each vehicle in year 2040 under each scenario to get each vehicle's failures per mile in 2040.

\section{Step 2 (Non-Vehicle Assets): Link Non-Vehicle Asset Ages with Failure Rates Using TCRP's Age-Based Model}

TCRP's Age-Based Model uses a Weibull distribution to calculate the probability of failure based on the age of nonvehicle transit assets. The TCRP report also provides shape and scale parameters based on national data for a range of asset types. Although there are 127 specific asset types listed in the TCRP report, we modeled only a subset which we believe will cause delay when failure occurs. These include guideway assets (31 categories, including tracks, viaducts, crossovers, tunnels, fills, and ballasts), systems assets (15 categories, including train controls, catenary, and signal systems), and electrification assets (8 categories, including third rails, power cables, and substations).

\section{Step 3 (Vehicles): Link Per Mile Failure Rates with Travel Delays}

TCRP Report 157 recommends using the following equation to calculate passenger delay per road call or vehicle failure:

$$
P D R=H\left(\frac{P M}{V M}+\frac{R T * P T}{V H}\right)
$$

where,

$$
\begin{aligned}
& P D R=\text { passenger delay per road call } \\
& H=\text { headway in minutes } \\
& P M=\text { passenger miles } \\
& V M=\text { revenue vehicle miles } \\
& R T=\text { recovery time } \\
& P T=\text { passenger trips } \\
& V H=\text { revenue vehicle hours }
\end{aligned}
$$


Data on passenger miles, vehicle miles, headways, and boardings for each operator were taken from Travel Model One's baseline 2040 projections. Equation 1 assumes that passengers on the failing vehicle and those waiting for the failing vehicle will be picked up by the next scheduled vehicle and, therefore, their delay is equal to headways. The average number of passengers on the bus or train is $\left(\frac{P M}{V M}\right)$. The number of people waiting for the broken vehicle along the route until a replacement bus or train takes over is $\left(\frac{R T * P T}{V H}\right)$. This second calculation is problematic for MTC's data since the number of buses and trains running likely is not distributed evenly throughout a day's worth of revenue vehicle hours. To account for this, we substituted equation 2 to calculate the number of people waiting for the failed vehicle. For this analysis, we assumed that recovery miles (the number of miles before another bus takes over the route) were equivalent to one-half the operator's average route length, but further research could improve this assumption.

$$
P W V=\left(\frac{P T}{V M}\right) * M R
$$

where,

$P W V=$ passengers waiting for the failed vehicle

$M R=$ recovery miles (miles before another bus takes over the route)

An added component of delay can occur in the case of rail failures when a failed train is blocking the passage of other trains. There is no TCRP equation to quantify this, so we used our own. If the average time to remove a blocking train is less than headways, there will be no delay arising from waiting behind a stalled train because the train will be cleared before the next train gets there. If this is not the case, equations 3,4 , and 5 can be used.

$$
\begin{aligned}
& D W B T=A W T *\left(\frac{P M}{V M}\right) \\
& A W T=\frac{\sum_{i-N T}\left(\frac{T C}{H}\right)-i}{N T} * H \\
& N T=\text { RoundDown }\left(\frac{T C}{H}\right)
\end{aligned}
$$

where,

$D W B T=$ delay from waiting behind stalled trains

$A W T=$ average wait time in headways for trains stuck behind stalled train

$i=$ each additional train

$T C=$ average time it takes to clear tracks

$N T=$ the number of trains that are delayed due to a stalled train ahead 
In equation 5, we rounded down the number of headways that pass during the time it takes to clear the tracks, because an additional train reaches the delay point only every full headway. The average time it takes to clear the tracks was information gathered from individual rail operators.

Another adaptation we made to TCRP's model of vehicle delay was to differentiate between two types of expected delay, which we call Type 1 Expected Delay and Type 2 Expected Delay. Expected delay is the chance of experiencing a failure multiplied by the delay that arises when a failure occurs. Expected delay is what we used as an input into Travel Model One.

Type 1 Expected Delay adds to in-vehicle travel time and was calculated per mile. Type 2 Expected Delay adds to out-of-vehicle travel time and was calculated per boarding. Both of these delay types were easily inserted into Travel Model One by adding a script to adjust skims and headways.

To calculate the two types of expected delay, we combined parts of the previous equations:

$$
\begin{aligned}
& T 1 E D(V)=R M *\left(D W B T+\left(H *\left(\frac{P M}{V M}\right)\right)\right) \\
& T 2 E D(V)=\frac{(H * P W V) *(R M * V M * 300)}{P T * 300}
\end{aligned}
$$

where,

$\operatorname{T1ED}(V)=$ Type 1 Expected Delay from vehicle failures

$R M=$ road calls per mile from Step 2 above

$T 2 E D(V)=$ Type 2 Expected Delay from vehicle failures

PBDV $=$ per boarding delay from vehicle failures (type 2 delay)

In equation 7, the numerator is composed of total passenger delay per boarding $(H * P W V)$ and the expected number of annual failures $(R M * P M * 300)$. This total annual delay is per annual boarding $(P T * 300)$. Miles and boardings were annualized using 300 instead of 365 to represent the fact that travel on weekends is expected to be less than travel on the typical weekday modeled by Travel Model One. This is consistent with other assessments used by MTC.

We adjusted equations 6 and 7 to cap the wait time on vehicles, behind stalled vehicles, and waiting for a failed vehicle at $\mathbf{3 0}$ minutes, since some average headways are longer than that. We assumed that after 30 minutes, a delayed passenger will either choose another mode (in some cases a replacement bus sent by the operator) to get to his/her destination or decide not to take the trip. Thus, we replace $H$ with $\operatorname{Min}(H, 30)$ in both equations.

\section{Step 3 (Non-Vehicle Assets): Link Probability of Failure with Travel Delays Using a New Operator-Informed Model}

For non-vehicle assets such as fixed guideways, train control systems, and electrification elements, there is no established model for translating non-vehicle transit asset failures 
into travel time delays. Based on discussions with BART and Caltrain staff, we developed a set of equations to quantify Type 1 and Type 2 Expected Delay, which is associated with the age of non-vehicle assets.

When a non-vehicle asset fails, three groups of riders potentially are affected: (1) those on vehicles affected by slow zones, (2) those on vehicles that have been stopped and cannot proceed until a non-vehicle failure has been addressed, and (3) those waiting to board a vehicle that has been stopped. Due to the potential for long repair times, we capped the wait time for groups (2) and (3) at 30 minutes, assuming that they will either switch modes or cancel their trip.

Type 1 Expected Delay includes delay experienced by riders affected by slow zones and by riders riding in a vehicle that has been stopped. Delay experienced by people waiting for a stopped vehicle contributes to Type 2 Expected Delay. Expected delay for those on trains affected by slow zones can be calculated using the following equations:

$$
\begin{aligned}
& S Z D=P F *\left(\frac{N T * M D}{V M * 300}\right) \\
& N T=\text { RoundDown }\left(\frac{(T R)-\left(\frac{1}{2} H\right)}{H}\right) * L A
\end{aligned}
$$

where,

$$
\begin{aligned}
& S Z D=\text { expected delay arising from slow zones } \\
& P F=\text { probability of failure in } 2040 \text { (from Step } 2 \text { above) } \\
& M D=\text { minutes of delay to the train caused by slow zone } \\
& T R=\text { time until repair or replacement of the failed asset in minutes } \\
& L A=\text { average number of lines affected by failure }
\end{aligned}
$$

Equation 9 assumes the average train is half a headway away from the location of the non-vehicle asset at the time it fails. Average minutes of delay resulting from a slow zone (MD), average time until repair or replacement (TR), and average number of lines affected by asset failure (LA) is information specific to each non-vehicle asset type and operator. Rough estimates were developed in consultation with operators based on each Bay Area rail system's unique characteristics; future efforts should collect and use statistical data on the real-world operational impacts of failures to supplement our baseline assumptions.

Expected delay for passengers on trains that must stop until a non-vehicle asset is repaired or replaced can be calculated using equation 10 . This is similar to the calculation for expected delay due to a slow zone (equation 8).

$$
S T D=P F *\left(\frac{N T *\left(\frac{T R}{2}\right)}{V M * 300}\right)
$$

where,

STD $=$ expected delay from being on a stopped train due to a non-vehicle asset failure ahead 
Equation 10 assumes that the average train has to wait half the total time it takes to repair or replace the asset. We capped $T R / 2$ at 30 minutes, assuming that if a vehicle is stopped beyond that time, people will off-board and choose a different route.

As stated above, Type 1 Expected Delay for non-vehicle assets (arising per mile, experienced in-vehicle) is the sum of expected delay arising from slow zones (equation 8 ) and expected delay arising from having to wait in a vehicle while a non-vehicle asset is repaired or replaced (equation 10 ).

$T 1 E D(N V)=S Z D+S T D$

where,

$\operatorname{T1ED}(N V)=$ Type 1 Expected Delay from non-vehicle asset failures

Type 2 Expected Delay (arising per boarding, experienced out-of-vehicle) is associated with waiting for vehicles that have been stopped until a failed asset is repaired or replaced. Type 2 Expected Delay can be calculated using equation 12.

$$
\begin{aligned}
& T 2 E D(N V)=P F \frac{W T * W N}{W B * 300} \\
& W T=T R-\left(\frac{1}{2} H\right) \\
& W N=B M *\left(\frac{1}{2} A R L\right) * \min (N T, D T) \\
& D T=L A\left(\frac{M O D}{H}\right)
\end{aligned}
$$

where,

WT = additional out-of-vehicle wait time when a vehicle is stopped by a non-vehicle asset failure

$W N=$ number of passengers waiting to board a vehicle stopped by a non-vehicle asset failure

$W B=$ average weekday boardings

$B M=$ average boardings per mile

$A R L=$ average route length

$D T$ = number of trains passing through affected area in one day

$N T$ = number of trains affected by failure (equation 9)

$M O D=$ minutes of operation daily (for example, this is 1080 minutes if trains run from $6 \mathrm{AM}$ to $12 \mathrm{AM}$ )

We capped WT at 30 minutes. We estimated the number of lines affected by failure for each asset type ( $L A)$ based on the number of lines using the average section of track for each operator and whether a failure of the specific asset type would affect travel in one or both directions. 
One other assumption was that operators spend the needed funding to get failed assets back into service. Because the cost of such emergency repairs is not already factored into the cost side of the $B / C$ equation (which is based on the scenario's funding level), it must be added in once it is known which assets are likely to fail. To do this, we assumed that the cost of emergency repair or replacement is roughly equal to the value of the asset. We then multiplied the probability of failure by the value of each asset and added that to the cost side of the $B / C$ equation.

After calculating the two types of expected delay for both vehicle and non-vehicle assets, we added them together to get for each operator a total amount of in-vehicle delay per mile (Type 1 Expected Delay) and a total amount of out-of-vehicle delay per boarding (Type 2 Expected Delay). These totals are used as inputs in Travel Model One.

\section{Step 4: Link Travel and Wait Time Delays to Benefits Using Travel Model One}

To input delays into Travel Model One, we manually adjusted in-vehicle and out-ofvehicle travel time skims. Type 1 Expected Delay was added to the in-vehicle travel time skims based on the distance traveled on each operator and mode. Type 2 Expected Delay was added to the out-of-vehicle time skims based on the number of boardings for each operator between each set of travel zones. Once transit travel time skims were adjusted, these new times influenced all travel choices made within the model, including auto ownership, activity choice, destination choice, mode choice, and route choice. Results of Travel Model One scenarios included miles traveled by mode, travel times, and travel costs. When compared to the baseline model run, these results can be used to calculate the full set of benefits included in the standard $B / C$ assessment. These benefits are based on the outputs of Travel Model One and include collisions, air pollution, noise, active transportation, travel costs, and travel times. Each benefit is valuated based on previous research by MTC and detailed in the "Plan Bay Area Draft Performance Assessment Report" (Metropolitan Transportation Commission 2013).

\section{Results}

\section{Scenarios and Costs}

We assessed two regional funding scenarios in comparison to a baseline scenario: a zero funding (OF) scenario and a zero regional funding (ORF) scenario. The baseline scenario is defined as the funding required to maintain the current transit capital backlog until the year 2040. The OF scenario examines conditions in 2040 if assets are allowed to degrade without any SGR investment. The ORF scenario-approximately $40 \%$ of the baseline scenario funding - examines the consequences of cutting all regional funding to transit SGR so that the only funds available are from FTA, bridge tolls, sales taxes, and bonds.

We intended to examine an additional scenario where transit backlog is completely paid down by 2040; however, the difference in delays between the baseline scenario and the improvement scenario was negligible. This is due to the fact that MTC's version of TERM-Lite prioritizes timely replacement of the assets most linked with delay in part by using a Transit Capital Priorities (TCP) score. This score also is used in regional funding decisions and places highest priority on replacement of revenue vehicles, which have the 
greatest capacity to create delay. While the baseline scenario includes enough funding for timely replacement of revenue vehicles, in a zero backlog scenario, the region is able to pay for timely replacement of all assets, including those that are not directly linked to delay (stations and facilities). Although these assets likely have an impact on passenger comfort and ridership, previous research has suggested that this impact is secondary to that of delay (Deakin et al. 2012).

The costs of the baseline scenario are $\$ 27$ billion over the 28-year planning period in 2013 dollars. The ORF scenario spends $\$ 11$ billion in the same period. Expected emergency replacement costs for assets that fail in the 0RF scenario is $\$ 1.1$ billion in comparison with baseline. Emergency replacements beyond baseline total $\$ 1.2$ billion in the $\mathrm{OF}$ scenario. Total costs for each scenario include the cost savings from decreasing SGR funding and cost expenditures on emergency replacements. Final costs for each scenario in comparison to baseline are $-\$ 617$ million annually for the ORF scenario and $-\$ 1,011$ million annually for the $0 \mathrm{~F}$ scenario.

\section{Benefits}

To assess benefits, we compared the outputs of Travel Model One under baseline, ORF, and $\mathrm{OF}$ scenarios. We used travel model outputs to calculate the following benefits experienced by the region's population in 2040: travel time savings for all modes; travel cost savings related to driving, auto ownership, and parking; air pollution reduction including PM2.5, $\mathrm{CO}_{2}$, and other pollutants; reductions in fatalities, injuries, and property damage due to collisions; active transport health benefits; and noise reduction. These benefits are monetized according to the values in Table 9 of the "Plan Bay Area Draft Performance Assessment Report" (Metropolitan Transportation Commission 2013).

Lower spending on transit SGR is linked with greater in-vehicle and out-of-vehicle delays. These delays cause a shift away from transit to driving, causing increased VMT. Transit ridership region-wide declined from 2.16 million daily trips to 2 million trips in the ORF scenario and to 1.8 million trips in the OF scenario. BART and Caltrain, the two largest rail systems in the Bay Area, experienced the largest decreases in ridership, likely due to the age of those systems' assets today and the other modal options available to the riders they tend to attract. Both transit delays and the negative externalities from increased VMT (including congestion, pollution, and collisions) are reflected in the total regional benefits. Table 1 shows the breakdown of regional benefits, with the greatest impacts coming from travel times. 
TABLE 1.

Regional Impacts Compared to Baseline Scenario

\begin{tabular}{|l|c|c|}
\hline & $\begin{array}{c}\text { Zero Regional } \\
\text { Funding }\end{array}$ & $\begin{array}{c}\text { Zero } \\
\text { Funding }\end{array}$ \\
\hline Funding Levels (annually, million \$) & -617 & $-1,011$ \\
\hline Average delay per boarding (min) & 0.1 & 0.5 \\
\hline Average delay per mile (min) & 0.1 & 0.7 \\
\hline VMT (annually, million) & 655 & 1,324 \\
\hline Ridership (daily trips on transit) & $-160,000$ & $-360,000$ \\
\hline Air pollutant reduction benefits (2040, million \$) & -28 & -56 \\
\hline Collisions, active transport, \& noise benefits (2040, million \$) & -114 & -244 \\
\hline Travel cost benefits (2040, million \$) & -474 & $-1,012$ \\
\hline Travel time benefits (2040, million \$) & $-1,004$ & $-1,493$ \\
\hline Total benefits (2040, million \$) & -1620 & -2806 \\
\hline Benefit/cost ratio & 2.6 & 2.8 \\
\hline
\end{tabular}

When we compared the total benefits and funding levels in Table 1 , we found a $B / C$ ratio of 2.8 for moving between a zero funding and baseline scenario. We found a $B / C$ ratio of 2.6 for moving between a scenario with zero regional funding and a baseline scenario. These ratios demonstrate diminishing returns to SGR investment. This is to be expected when operators prioritize replacement of assets linked to the greatest user benefits.

\section{Conclusions}

We found that current SGR funding levels compared to scenarios where funding is reduced generates a benefit/cost ratio of between 2.6 and 2.8 over the 28-year planning period, which is a very conservative estimate. "Plan Bay Area Draft Performance Assessment" (Metropolitan Transportation Commission 2013), which uses a parallel methodology to assess new transit infrastructure projects, found that transit efficiency projects, such as frequency and speed enhancements to existing transit services, generate an average benefit/cost ratio of 1.4 when weighted by size. Transit expansion projects, such as rail extensions and bus rapid transit corridors, generate an average benefit/cost ratio of 2.8 when weighted by size. From these numbers, we can conclude that SGR funding should, indeed, be a high-ranking regional priority.

The benefit/cost ratio here is for all 25 transit systems together. However, SGR funding likely has much higher benefits for systems with higher ridership.

Whereas it is clear that current funding levels for transit SGR have societal benefits that far exceed their costs, the change in delays and slope of the benefit/cost curve along different funding scenarios indicate diminishing returns. This implies that, at some point, increasing funding for transit SGR is not economically efficient. Testing more scenarios would help to indicate where this point lies. Our inability to show travel time benefits when moving from current funding levels to a state of zero backlog suggests that it is possible that the Bay Area has either reached or exceeded this point. Additionally, the lack of delay resulting from current funding and prioritization algorithms also indicates 
that transit operators already are maximizing benefits to society through their judicious use of limited funding.

\section{Recommendations and Future Research}

Based on this research, it is recommended that transit operators in the Bay Area continue to prioritize vehicles and other high-impact assets for SGR funding, because this prioritization mitigates the majority of delays associated with baseline funding as compared to fully-funded SGR. It also is recommended that MTC as a regional agency continues to fund transit SGR, given the fairly high $\mathrm{B} / \mathrm{C}$ ratio of regional funding (2.6). However, it is recommended that additional funding for transit be used for expansion rather than SGR, because the benefits of SGR funding rapidly diminish at levels higher than the current baseline.

Future research should expand to other regions within the United States and worldwide. Bay Area research on SGR should increase in specificity, comparing the benefits of SGR funding for different transit systems within the Bay Area. Additionally, future research should begin to address the limitations of the current study. Specifically, these limitations include our inability to model the impact of degradation for a large set of assets not directly linked with delay, such as stations and facilities. These nonoperational impacts, such as user comfort or perceived security, certainly affect modal choice decisions. Finally, future research should confirm estimates of failure recovery times, rail lines affected by non-vehicle asset failures, slow zone speed restrictions, and additional delay to excessive failures and staff constraints in very degraded scenarios.

\section{References}

Cohen, Harry. 2012. "Relationship of Asset Condition Rating to Transit System Performance," December, http://onlinepubs.trb.org/onlinepubs/reports/TERM_ March_2013Cohen.pdf.

Deakin, Elizabeth, Arlee Reno, James Ruin, Sean Randolph, and Michael Cunningham. 2012. "A State of Good Repair for BART: Regional Impacts Study: What Could Happen if BART Fails to Maintain a State of Good Repair." http://www. bayareaeconomy.org/media/files/pdf/BART_SGR_-_Regional_Impacts___Final_ Report_May_2012.pdf.

Federal Transit Administration. 2007. "Useful Life of Transit Buses and Vans." Report No. FTA VA-26-7229-07.1, http://www.fta.dot.gov/documents/Useful_Life_of_Buses_ Final_Report_4-26-07_rv1.pdf.

Metropolitan Transportation Commission. 2013. "Plan Bay Area: Strategy for A Sustainable Region." http://files.mtc.ca.gov.s3.amazonaws.com/pdf/Plan_Bay_Area_ FINAL/pbafinal/index.html.

Metropolitan Transportation Commission. 2013. "Plan Bay Area Performance Assessment Report." http://planbayarea.org/pdf/Draft_Plan_Bay_Area/ Performance_Report.pdf. 
Transportation Research Board. 2012. "State of Good Repair: Prioritizing the Rehabilitation and Replacement of Existing Capital Assets and Evaluating the Implications for Transit." TCRP Report 157, http://onlinepubs.trb.org/onlinepubs/ tcrp/tcrp_rpt_157.pdf.

United States Government Accountability Office (U.S. GAO). 2013. "Transit Asset Management: Additional Research on Capital Investment Effects Could Help Transit Agencies Optimize Funding." www.gao.gov/products/gao-13-571.

Voith, Righard P., Peter A. Angelides, and Adam Ozimek. 2013. "The Economic Value of Transit and the Effect of Insufficient Capital Funding - A Case Study of the Southeastern Pennsylvania Transportation Authority (SEPTA)." Transportation Research Board Annual Meeting 2014, Paper \#14-558.

\section{About the Authors}

Liz PATERSon (liz.paterson@unhabitat.org)works for the United Nations Human Settlements Programme (UN-Habitat) in the Urban Economy Branch where she consults on transportation, planning, and economic development projects in growing cities internationally. She coordinates the economy and finance components of sustainable development projects in Colombia, Egypt, Mozambique, Philippines, Rwanda, and other countries and also develops spatial-economic assessment tools for use in data-poor environments. She formerly worked for Metropolitan Transportation Commission in the San Francisco Bay Area. She holds Master of Urban and Regional Planning and Master of Public Health degrees from Portland State University.

DAVID VAUTIN (dvautin@mtc.ca.gov) is a Senior Transportation Planner at the Metropolitan Transportation Commission (MTC) in Oakland, California, leading the agency's efforts in the fields of performance assessment and performance monitoring. His analytical work informs regional policy decisions by monitoring adherence to adopted goals and targets and by identifying high-performing transportation investments that achieve the region's sustainability objectives. He holds a Master of Science degree in Civil Engineering from the University of California, Berkeley and a Bachelor of Science degree in Civil Engineering from Cornell University. 